\title{
Webinar „Antikoagulation“
}

\section{Universitätsspital Lausanne (CHUV), Klinik für Angiologie}

\section{J. Hafner, Ph. Kern, L. Mazzolai}

Am 10. September führte die Schweizerische Gesellschaft für Phlebologie unter der fachlichen Leitung von Philippe Kern, Jürg Hafner und Lucia Mazzolai (Prof. Dr. med., Chefärztin Angiologie) am Universitätsspital Lausanne (CHUV) ein Webinar zum Thema „Antikoagulation“ durch:

- Direkte orale Antikoagulation bei Patienten mit einem malignen Tumor (Adriano Alatri, Lausanne)

- Direkte orale Antikoagulantien - Indikationen, Anwendung, Monitoring (Jan-Dirk Studt, Zürich)

- Superfizielle Venenthrombose (Brigitte Ney, Lausanne)

- Venöse Thromboembolien: Wie lange antikoagulieren, hilft das Thrombophilie-Labor? (Jan-Dirk Studt, Zürich)

- Venöse Thromboembolie, Kontrazeption und Schwangerschaft: Update (Lucia Mazzolai, Lausanne)

- Antiphospholipid-Syndrom: Diagnostik, Labormonitoring, Behandlung (Lorenzo Alberio, Lausanne)

- Venöse Thrombosen in atypischer Lokalisation (Jan-Dirk Studt, Zürich)

Alle Vorträge waren von ausgezeichneter inhaltlicher und didaktischer Qualität. Die Organisatoren möchten den Referentinnen und Referenten ihren großen Dank aussprechen.

\section{Weiterverwendung auf der SGP-Teaching- Plattform}

„www.swissphlebology-teaching-webinars. org"

Jedes Jahr führt die Union der Schweizerischen Gesellschaften für Gefässkrankheiten (USGG) im Rahmen der schriftlichen Prüfung zum Facharzttitel in Angiologie auch gleichzeitig die Prüfung zum Fähigkeitsausweis in Phlebologie (USGG/SIWF) im Multiple-Choice(MC)-Format durch. Leider scheitern Jahr für Jahr erstaunlich viele Prüfungsteilnehmer*innen an dieser Prüfung.

Diese ersten 7 und in Zukunft auch noch viele weitere Vorträge sollen die Prüfungskandidat*innen wirksam bei der Prüfungsvorbereitung unterstützen. Um Zutritt zum Teaching Webinar zu erhalten, melden die Kandidat*innen ihre Mitgliedschaft bei der Schweizerischen Gesellschaft für Phlebologie (SGP/SSP) an. So profitieren zukünftige Phlebolog*innen vom in Hülle und Fülle vorhandenen phlebologischen Fachwissen in der Schweiz, das jedoch nicht immer so leicht zugänglich ist. Dafür verpflichten sie sich zur aktiven Teilnahme am Gesellschaftsleben der SGP/SSP.

Alle 5 Referentinnen und Referenten haben dankenswerterweise ihre Zusage erteilt, dass ihr Vortrag für Lernzwecke auf einer passwortgeschützten Website zur Verfügung gestellt werden darf.

\section{Zusammenfassung des Vortrags: Antiphospholipid-Syndrom (Prof. Lorenzo Alberio, Lausanne):}

Das Antiphospholipidsyndrom (APS) ist durch die Klinik einerseits und seine Laborkriterien andererseits definiert.

Die Klinik umfasst (A) entweder thrombotische Ereignisse (venöse Thromboembolie und/oder arterielle Thrombosen und/oder Mikrothrombosen) und/oder (B) Schwangerschaftskomplikationen, das heißt mindestens drei wiederholte idiopathische Aborte vor der zehnten Schwangerschaftswoche oder einen fötalen Tod im 2./3. Trimenon oder eine schwere Präeklampsie.
Die Laborkriterien umfassen den Nachweis von drei unterschiedlichen Antikörpern (AK), die alle drei gegen unterschiedliche Epitope des beta2-Glykoproteins-I gerichtet sind, das seinerseits wieder an der Zellmembran von Endothelzellen und Blutplättchen exprimiert wird: (A) dem Lupus-Antikoagulans (klinisch am wichtigsten), (B) der Anticardiolipin-AK und (C) dem Screening-Test für Anti-beta2-Glykoprotein I-AK. Die drei Tests müssen über eine Periode von 12 Wochen positiv bleiben, damit das Resultat gültig ist [J Thromb Haemost 2006; 4: 295]. Im Serum zirkulieren nicht immunogene Monomere des beta2-Glykoproteins-I, die erst als membranständige Dimere immunogen werden können. APL-AK-Ablagerungen an den Gefäßendothelien aktivieren Komplement, das neutrophile Granulozyten bindet und die „Neutrophil Extracellular Traps“ auslöst (NETose), ein in sich stark thrombogener Prozess. Hinzu kommen die Plättchenaktivierung mit Freisetzung von Thromboxan A2 und Glykoprotein IIb/III sowie generell die Freisetzung des Gewebefaktors in erster Linie aus dem Gefäßendothel. Alle diese biochemischen Prozesse münden in eine lokale Gefässthrombose. Meistens sind ein biologischer „Second Hit“ wie eine schwere Entzündung mit CRP-Anstieg oder ein hormoneller Einfluss (Östrogenzufuhr) im Spiel. Die Schwangerschaftskomplikationen entstehen hingegen nicht etwa durch planzentare Mikrothrombosen, sondern durch eine Trophoblasten-Atrophie, die durch die Autoantikörper verursacht wird [Lancet 2010; 376: 1498. CMAJ 2003; 168: 1675. N Engl J Med 2018; 378: 2010].

Das Antiphospholipid-AK-Paradox: In vitro verlängern Antiphospholipid-AK die Gerinnungstests aPTT und dRVVT, was auf den ersten Blick auf eine antikoagulatorische Wirkung in vivo hinweisen könnte. Das 
Gegenteil ist jedoch der Fall (siehe oben). In vitro lagern sich die APL-AK an den Prothrombinase-Komplex (Xa+Va+Calcium) an und behindern so die Aktivierung der weiteren Gerinnungskaskade. Dadurch verlängern sich die aPPT und dRVVT in vitro.

Der Lupus-Antikoagulanstest läuft über drei Stufen (Screening, Mischung von Seren und Bestätigung). Das Labor gibt alle drei Teststufen sowie eine Schlussbeurteilung ab. Patienten unter DOAC, Vitamin-K-Antagonisten (VKA) oder niedermolekularem Heparin $(\mathrm{NMH})$ haben oft falsch positive Tests. Daher ist es sehr wichtig, dass die Einsender dem Labor stets mitteilen, ob der Testpatient unter irgendeiner Form von Antikoagulation steht [Curr Rheumatol Rep 2020; 22: 38]. Falls verantwortbar, sollte die Antikoagulation 1-2 Tage vor dem Test unterbrochen werden. Auch während einer Schwangerschaft oder bei Akutphasen-Entzündungen mit erhöhtem CRP kommen falsch positive oder falsch negative Testresultate gehäuft vor. Die beiden anderen Tests (anti-Cardiolipin, anti-beta2-Glykoprotein-I) basieren auf Festphasenbiochemie (ELISA). Da die Resultate zwischen den Labors schlecht vergleichbar sind, sollten die 99.-Perzentile-Werte herangezogen und im Verlauf sollte immer dasselbe Labor verwendet werden [Curr Rheumatol Rep 2020; 22: 38].

Test-Interpretation: Das Lupus-Antikoagulans ist von den drei Tests der stärkste Risikofaktor für thromboembolische vaskuläre Ereignisse und Schwangerschaftskomplikationen. Insbesondere eine dreifache Positivität für alle drei Tests geht mit einem hochsignifikanten Risiko für thromboembolische Ereignisse und für Schwangerschaftskomplikationen einher. Wenn die beiden Festphasentests positiv anzeigen, ist das Resultat valider, wenn zweimal IgG oder zweimal IgM positiv ist (gleiches Isotop positiv). IgG-Antikörper sind klinisch relevanter als IgM-Antikörper. Die Kombination von IgG-anti-Cardiolipin-AK und Anti-beta2-Glykoprotein-I-AK ist mit einem signifikant höheren Risiko für venöse Thromboembolie und Schwangerschaftskomplikationen verbunden. Doppelte IgM-Positivität ist mit Schwangerschaftskomplikationen assoziiert. Alle übrigen Konstellationen haben keine signifikante Häufung thrombotischer Ereignisse oder von Schwangerschaftskomplikationen zur Folge [Thromb Haemost 2005; 93: 1147. N Engl J Med 2018; 378: 2010].

Primäre Prävention: Patienten mit einem bekannten Antiphospholipidsyndrom erhalten in Situationen mit erhöhtem Thromboembolierisiko, etwa im Rahmen großer chirurgischer Eingriffe, post partum oder bei langen Flugreisen, niedermolekulares Heparin (NMH) [Lupus 2011; 20: 2016]. Patientinnen mit einem Antiphospholipidsyndrom im Rahmen eines systemischen Lupus erythematodes erhalten Low-dose-Aspirin kombiniert mit Hydroxychloroquin und in Risikosituationen zusätzlich ein NMH [Lupus 2012; 21: 751. Blood 2009; 114: 2020].

Therapie und sekundäre Prävention: Die Therapie und die sekundäre Prävention bauen praktisch gänzlich auf VitaminK-Antagonisten (VKA) auf (Ziel-INR 2-3) [NEJM 2003; 349: 1133]. Arterielle Thromboembolien profitieren vermutlich vom Zusatz von Low-dose-Aspirin [Ann Rheumat Dis 2019;70:1296]. Der ischämische Stroke erfordert ebenfalls Low-dose-Aspirin + VKA [JAMA 2004; 291: 576. Blood 2009; 114: 2020]. Schwangerschaftskomplikationen werden mit einer Kombination aus Low-
dose-Aspirin und NMH behandelt [Cochrane Database Syst Rev 2005: CD002859]. Vor dem Beginn einer oralen Antikoagulation mit VKA sollte - wenn es die klinische Situation erlaubt - der blande INR-Wert ohne Antikoagulation bestimmt werden. In Gegenwart von Antiphospholipid-AK kann dieser Vorwert ohne Therapie bereits über einem INR von 1,0 liegen, was natürlich das INR-Monitoring unter Therapie beeinflusst [Lancet Haematol 2020; 7e: 613].

Direkte orale Antikoagulanzien haben bei Tripel-Positivität oder nach einer durchgemachten arteriellen Thrombose keinen Platz in der Behandlung des Antiphospholipidsyndroms [Blood 2018; 8: 237]. Nach durchgemachten unkomplizierten tiefen Beinvenenthrombosen sind sie einer VKATherapie nicht unterlegen [Lancet Haematol 2016; 3: e426. Ann Rheumat Dis 2019; 78: 1296].

Bei besonders dramatisch verlaufenden, therapierefraktären Antiphospholipidsyndromen kann die Intensität der oralen Antikaogulation mit Vitamin-K-Antagonisten erhöht werden, die Heparintherapie kann intensiviert oder es kann auf Fondaparinux umgestellt sowie Clopidogrel hinzugegeben werden. Schließlich kommen Plamaaustauschverfahren und/oder eine B-ZellDepletierung mit Rituximab zum Einsatz [Lancet Haematol 2020; 7: e613].

\section{IMPRESSUM}

Verantwortlich für Mitteilungen der SGP:

Prof. Dr. med. Jürg Hafner, Zürich 